\title{
Association between inflammation-based prognostic scores and in-hospital outcomes in elderly patients with acute myocardial infarction
}

This article was published in the following Dove Press journal:

Clinical Interventions in Aging

\author{
Rui Wang' \\ Xiaodan Wen ${ }^{2}$ \\ Cheng Huang' \\ Yingcong Liang' \\ Yujing Mo' \\ Ling Xue'
}

'Department of Cardiology, Guangdong Cardiovascular Institute, Guangdong

Provincial People's Hospital, Guangdong Academy of Medical Sciences,

Guangzhou, People's Republic of China; ${ }^{2}$ Department of Geriatrics, Guangdong Geriatrics Institute, Guangdong

Provincial People's Hospital, Guangdong Academy of Medical Sciences,

Guangzhou, People's Republic of China

Correspondence: Ling Xue

Department of Cardiology, Guangdong

Cardiovascular Institute, Guangdong

Provincial People's Hospital, Guangdong

Academy of Medical Sciences, Zhongshan

No. 2 Road \#I06, Yuexiu District,

Guangzhou 510080, People's Republic of

China

$\mathrm{Tel} / \mathrm{Fax}+8620838278$ I2I 0620

Email drxueling@126.com
Objective: Emerging evidence suggests that systemic inflammation is a predictor of poor prognosis in acute myocardial infarction (AMI). In this study, we sought to assess whether inflammation-based prognostic scores are associated with in-hospital outcomes in elderly patients with AMI.

Methods: In this retrospective study, patients who were over 75-years-old and met the diagnostic criteria for AMI were consecutively recruited from January 1, 2016, to March 31, 2019. Logistic regression and receiver-operating characteristic (ROC) analyses were performed to evaluate the predictive value of the inflammation-based Glasgow Prognostic Score (GPS), Prognostic Index (PI) and Prognostic Nutritional Index (PNI).

Results: A total of 273 patients were enrolled. The incidence of major cardiovascular adverse events (MACEs) and mortality during hospitalization increased significantly with increasing GPS and PI scores. Multiple logistic regression showed that the GPS was independently associated with MACEs (score 1, RR: 6.711, 95\% CI: 1.409-31.968; score 2, RR: 14.063, 95\% CI: 3.018-65.535) and mortality (score 1, RR: 8.656, 95\% CI: 1.068 70.126; score 2, RR: 10.549, 95\% CI: 1.317-84.465). The PI was also independently predictive of MACEs (score 2, RR: 5.132, 95\% CI: 1.451-18.148). No significant difference was observed in the PNI between patients with different in-hospital outcomes. When inhospital MACEs were used as an endpoint, the area under the curve (AUC) of the GPS was 0.740 (95\% CI 0.678-0.802), and the AUC of the PI was 0.703 (95\% CI 0.634-0.773). When mortality was used as an endpoint, the AUC of the GPS was 0.677 (95\% CI 0.602-0.753), and the AUC of the PI was 0.667 (95\% CI 0.577-0.757).

Conclusion: The severity of systemic inflammation is a strong predictor of poor prognosis in elderly patients with AMI. Among these three inflammation-based prognostic scores, the GPS has a better predictive value than the PI and PNI for in-hospital MACEs and mortality. Keywords: inflammation-based prognostic scores, acute myocardial infarction, elderly patients

\section{Introduction}

Despite the extensive development of percutaneous coronary intervention (PCI) and other revascularization strategies, acute myocardial infarction (AMI) remains one of the leading causes of death worldwide. ${ }^{1}$ Numerous risk factors have been shown to be associated with mortality in AMI. Among these factors, systemic inflammation is gradually becoming recognized as a strong predictor of poor prognosis. Maarten Vanhaverbeke et al demonstrated that peak C-reactive protein (CRP) is an independent predictor of left ventricular dysfunction after acute myocardial infarction 
(AMI). ${ }^{2}$ Recently, Yu Jia et al found a good correlation between the baseline inflammation-based Glasgow Prognostic Score (GPS) and the mortality of ST-segment elevation myocardial infarction (STEMI) ${ }^{3}$. However, few data are available on the relationship between inflammation and AMI prognosis in elderly patients. Frailty and malnutrition are common in the elderly population, ${ }^{4,5}$ and previous studies suggested that these two conditions could also lead to changes in the inflammation status. ${ }^{6,7}$ Thus, it is unclear whether the distinct spectra of inflammatory biomarkers in older people influence the predictive value of inflammation in AMI.

Several inflammation-based prognostic scores have been well established to date, which were initially used to predict the outcomes of cancer patients. As mentioned above, the inflammation-based Glasgow Prognostic Score (GPS), which is composed of serum CRP and albumin, has been shown to be associated with mortality in many malignant diseases. ${ }^{8}$ The Prognostic Index (PI), which is composed of serum CRP and the peripheral WBC count, was also reported to be a strong predictor for survival in advanced lung cancer patients. ${ }^{9}$ The Prognostic Nutritional Index (PNI), which is based on serum albumin and the peripheral lymphocyte count, is an independent predictor of poor outcome in patients with hepatocellular carcinoma. $^{10}$

In this study, we sought to use these three inflammation-based scores to evaluate the severity of inflammation in elderly patients with AMI and to assess whether these scores are associated with in-hospital cardiovascular adverse events and all-cause mortality.

\section{Methods}

\section{Subjects}

In this retrospective cohort study, patients were enrolled from the Coronary Care Unit (CCU) of Guangdong Provincial People's Hospital from January 1, 2016, to March 31, 2019. Consecutive patients who were over 75years-old and met the Third Universal Definition of Myocardial Infarction ${ }^{11}$ were recruited. The exclusion criteria for this study included the following: 1) lack of serum CRP, albumin, peripheral white cell count (WBC) and lymphocyte count; and 2) refusal to participate in this study. Finally, a total of 273 elderly patients with AMI were enrolled in this study (Figure 1). Written informed consent was obtained from all patients. This study was approved by the Ethics Committee of Guangdong Provincial People's Hospital.

\section{Data collection}

Baseline data included demographic characteristics, history of hypertension, diabetes, smoking, history of myocardial infarction or PCI, symptom-onset-to-balloon time (S2B), and type of AMI (STEMI or NSTEMI). At admission, we recorded the patient's Killip class, heart rate,

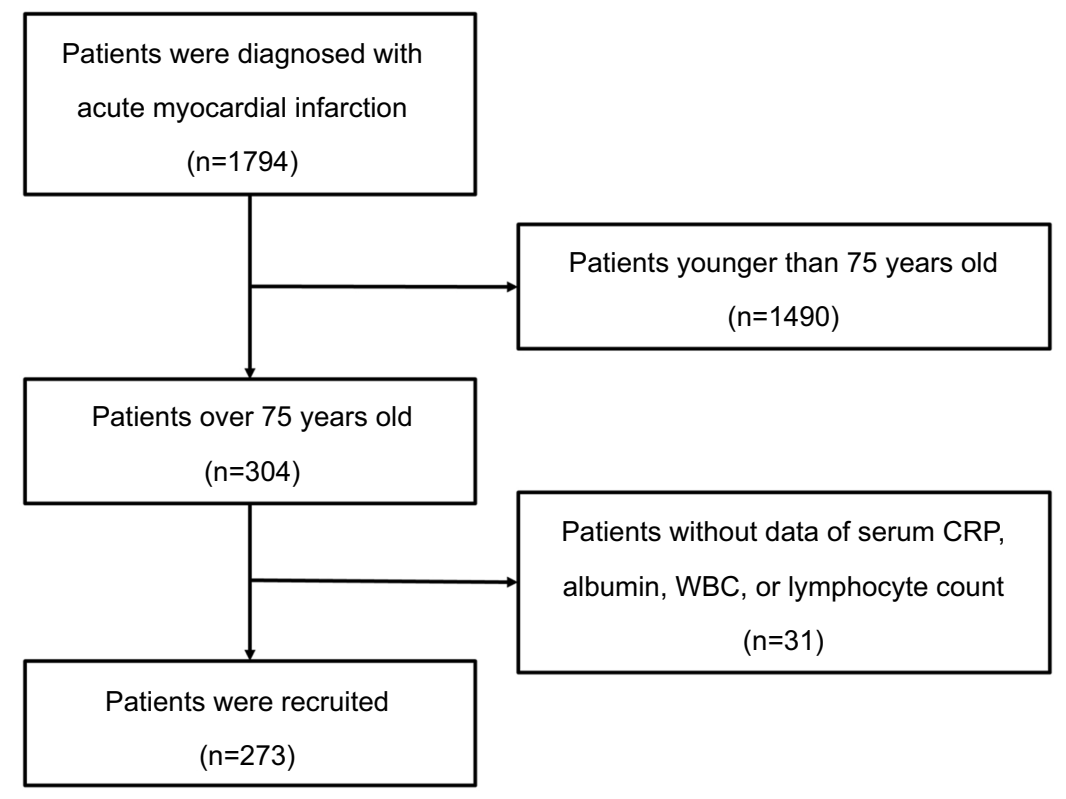

Figure I Flowchart of the patient selection process.

Abbreviations: CRP, C-reactive protein; WBC, white blood cell. 
blood pressure, and left ventricular ejection fraction (LVEF), as measured by echocardiography with Simpson's method. All the lab tests for blood samples were run in real time for clinical purposes and were performed in the laboratory department (in accordance with the ISO 9000 Quality Management and Assurance Standards) at our medical centre with standard examination methods. The results of lab tests, including routine blood tests, serum CRP and albumin, were also collected at admission.

Data on in-hospital major cardiovascular adverse events (including cardiovascular death, cardiac shock, mechanical complications of myocardial infarction, cerebral infarction, and recurrent $\mathrm{MI}$ ) and all-cause mortality were collected from the electronic medical history system. The primary endpoint of this study was the composite endpoint of inhospital major cardiovascular adverse events (MACEs), and the secondary endpoint was in-hospital all-cause mortality. The constituents of the three inflammation-based prognostic scores (GPS, PI and PNI) are listed in Table 1.

\section{Statistics}

Univariate analyses of normally distributed continuous variables are expressed as the mean $\pm \mathrm{SD}$ and were performed

Table I Inflammation-based prognostic scores

\begin{tabular}{|c|c|c|}
\hline \multicolumn{2}{|c|}{ Scoring system } & Score \\
\hline \multicolumn{2}{|c|}{ Glasgow Prognostic Score (GPS) } & \\
\hline CRP & $\begin{array}{l}>10 \mathrm{mg} / \mathrm{L} \\
\leq 10\end{array}$ & $\begin{array}{l}1 \\
0\end{array}$ \\
\hline serum albumin & $\begin{array}{l}<35 \mathrm{~g} / \mathrm{L} \\
\geq 35 \mathrm{~g} / \mathrm{L}\end{array}$ & $\begin{array}{l}\text { I } \\
0 \\
\text { Total score }\end{array}$ \\
\hline \multicolumn{2}{|c|}{ The prognostic index $(\mathrm{PI})$} & \\
\hline CRP & $\begin{array}{l}>10 \mathrm{mg} / \mathrm{L} \\
\leq 10\end{array}$ & $\begin{array}{l}1 \\
0\end{array}$ \\
\hline WBC & $\begin{array}{l}>11 * 10^{9} / \mathrm{L} \\
\leq 11 * 10^{9} / \mathrm{L}\end{array}$ & $\begin{array}{l}\text { I } \\
0 \\
\text { Total score }\end{array}$ \\
\hline \multicolumn{2}{|c|}{ The prognostic nutritional index (PNI) } & \\
\hline \multicolumn{2}{|c|}{$\begin{array}{l}10 * \text { serum albumin }(g / L)+0.005 * \text { peripheral lympho- } \\
\text { cyte count }(/ \mathrm{uL}) \geq 45 \\
10 * \text { serum albumin }(g / L)+0.005 * \text { peripheral lympho- } \\
\text { cyte count }(/ \mathrm{uL})<45\end{array}$} & $\begin{array}{l}0 \\
\text { I } \\
\text { Total score }\end{array}$ \\
\hline
\end{tabular}

Note: The indicator $(*)$ represents multiplication.

Abbreviations: CRP, C-reactive protein; WBC, white blood cell. using one-way ANOVA for comparisons between survival/ death groups and no-MACEs/MACEs groups. A logarithmic transformation was performed for variables that were positively skewed. Variance was determined to be uneven by using the Welch test. Non-normally distributed continuous variables are presented as the median and interquartile range and were analysed using the Mann-Whitney $U$ test. The Pearson chisquare or Fisher exact tests were used, as appropriate, for categorical data, which are expressed as percentages.

Univariate/multivariate associations between clinical variables and in-hospital endpoints were estimated by logistic regression analysis with a forward stepwise regression model. Clinical variables that were significant at $P<0.05$ in the univariate analysis, along with clinically important factors, were included in the multivariate analysis. Receiver-operating characteristic (ROC) analysis was performed, and the area under the curve (AUC) was calculated to determine the predictive power of the prognostic scores for the in-hospital endpoints.

Data analyses were performed using SPSS 22.0 (SPSS, Inc., Chicago, IL), and MedCalc Version 19 (MedCalc Software, Ostend, Belgium) was used for the comparison of ROC curves. A two-sided $P$-value $<0.05$ was considered statistically significant.

\section{Results Baseline characteristics}

A total of 273 patients were enrolled in this study. The mean age was $81.2 \pm 4.2$-years-old, and $37.7 \%$ of the patients were female. A total of 67 (24.5\%) patients had MACEs, and 41 (15.0\%) patients died during hospitalization. The baseline demographic and clinical characteristics of the study are given in Table 2. Significant differences were observed in the Killip class, time from AMI to PCI, LVEF, heart rate, systolic blood pressure (SBP), diastolic blood pressure (DBP), WBC, neutrophils, haemoglobin, serum creatinine, serum albumin, N-terminal B-type natriuretic peptide (NT-proBNP), GPS and PI between patients with MACEs and without MACEs during hospitalization. Similarly, patients who died during hospitalization had a lower LVEF, higher Killip class, longer symptom-onset-to-balloon time, higher heart rate, lower systolic blood pressure, higher WBC, higher neutrophils, lower haemoglobin, higher serum creatinine, lower serum albumin, higher NTproBNP, higher GPS and PI than those in patients who did not die during hospitalization. 


\begin{tabular}{|c|c|c|c|c|}
\hline 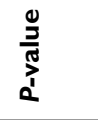 & \multicolumn{2}{|l|}{ 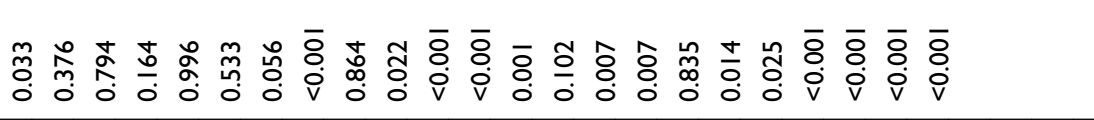 } & \multicolumn{2}{|c|}{$\begin{array}{l}\overline{0} \\
\end{array}$} \\
\hline 壱 & 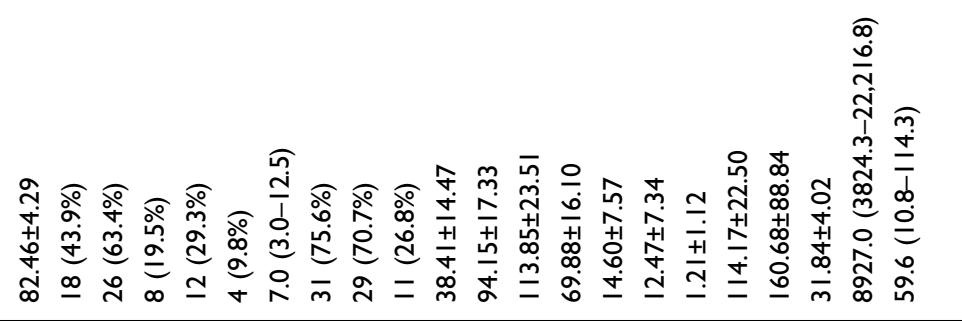 & 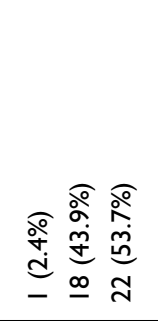 & 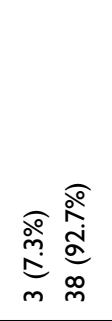 & 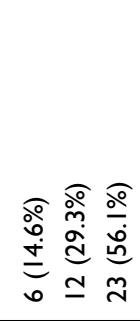 \\
\hline 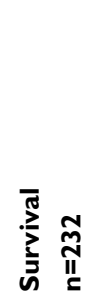 & 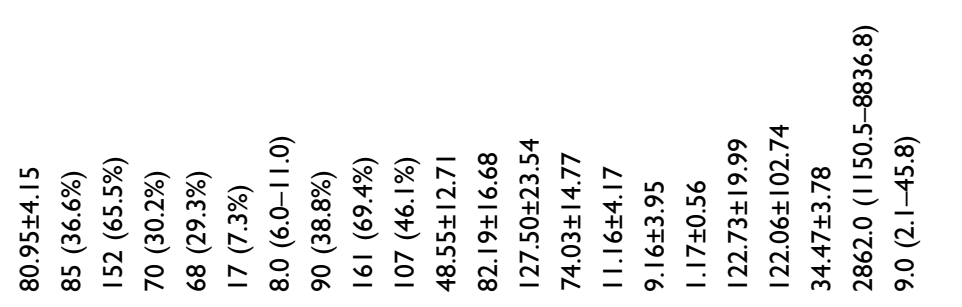 & 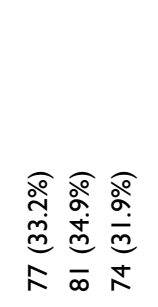 & 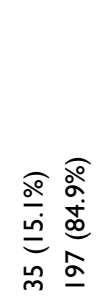 & 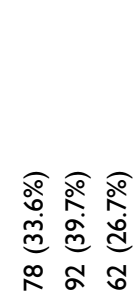 \\
\hline$\frac{0}{\frac{0}{2}}$ & 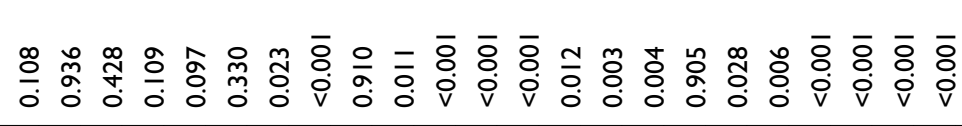 & & & \\
\hline 岂 & 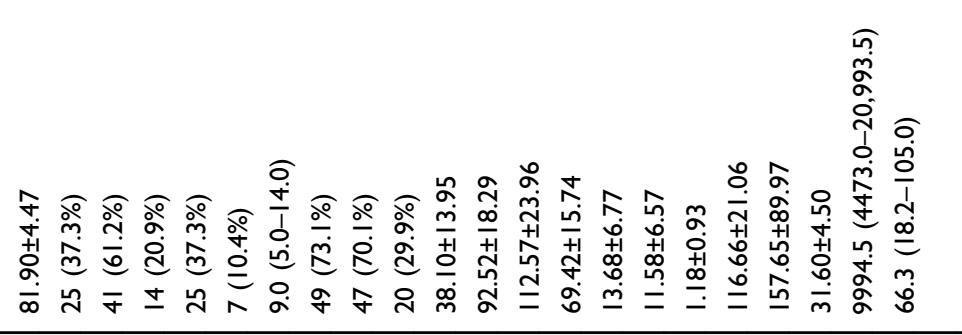 & 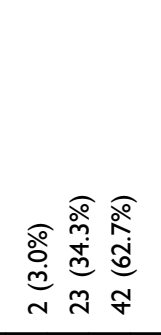 & 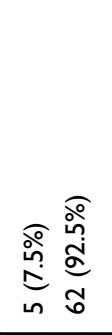 & 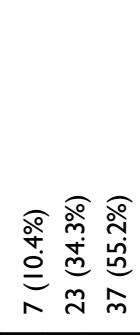 \\
\hline 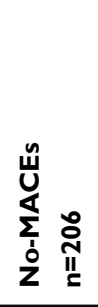 & 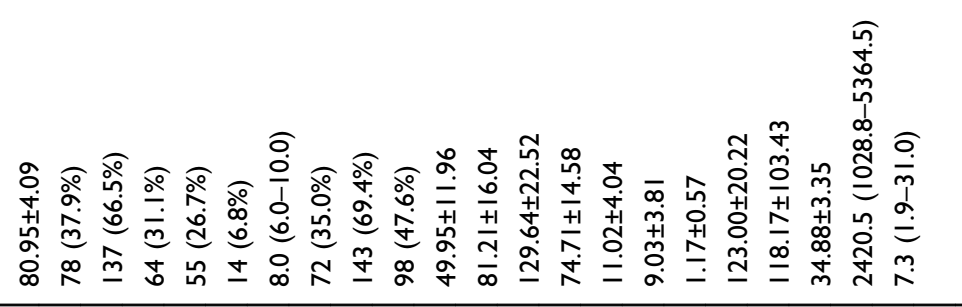 & 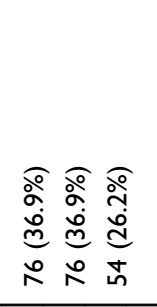 & 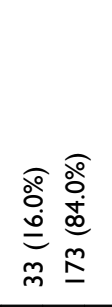 & 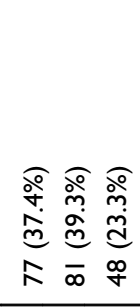 \\
\hline 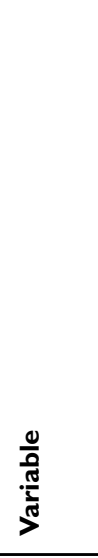 & 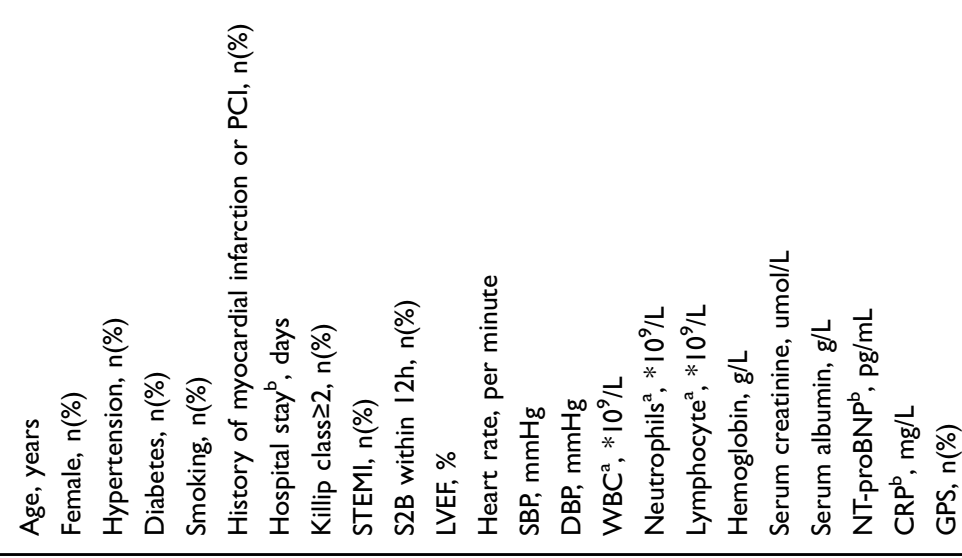 & $-r$ & & $0-4$ \\
\hline
\end{tabular}




\section{Association between inflammation-based \\ prognostic scores and in-hospital outcome}

The presence of MACEs and mortality during hospitalization increased significantly with increasing GPS and PI scores. There were no significant differences in the PNI between the patients with different in-hospital endpoints (Table 2).

Clinical variables that were significant at $P<0.05$ in the univariate analysis were included in the multivariate analysis, and since peripheral WBC and lymphocyte count, serum albumin and CRP were components of the inflammation-based prognostic scores, we did not include these variables in the multivariate regression model. After adjusting for confounding factors, such as age, heart rate, blood pressure, LVEF, peripheral haemoglobin concentration, serum creatinine, NT-proBNP, Killip class and symptom-onset-to-balloon time, multiple logistic regression analysis identified GPS as being independently associated with MACEs (score 1, RR: 6.711, 95\% CI: 1.409-31.968, $P=0.017$; score 2, RR: 14.063 , 95\% CI: 3.018-65.535, $P=0.001$ ) and in-hospital mortality (score 1, RR: 8.656, 95\% CI: $1.068-70.126, P=0.043$; score 2, RR: 10.549 , 95\% CI: $1.317-84.465, P=0.026)$. Increased PI values were also independently predictive of in-hospital MACEs (score 2, RR: 5.132, 95\% CI: 1.451-18.148, $P=0.011$ ), but there was no significant difference in mortality between patients with different PIs in the multiple regression model (Tables 3 and 4).

\section{Comparison of the prognostic values of inflammation-based scores}

In the ROC curve analyses, when in-hospital MACEs were used as a composite endpoint, the AUC of the GPS was 0.740 (95\% CI $0.678-0.802, P<0.001)$, and the AUC of the PI was 0.703 (95\% CI $0.634-0.773$, $P<0.001$ ) (Figure 2). When in-hospital mortality was used as an endpoint, the AUC of the GPS was 0.677 (95\% CI 0.602-0.753, $P<0.001$ ), and the AUC of the PI was 0.667 (95\% CI $0.577-0.757, P=0.001$ ) (Figure 3). When the AUC values were compared among groups, the GPS showed a higher distinguishing power than PI for predicting in-hospital MACEs and mortality (MACEs: $P<0.001$, mortality: $P=0.001$ ) (Table 5). Similar to the univariate analysis, in the ROC curve analyses, PNI could not predict in-hospital MACEs or mortality (MACEs: $P=0.293$, mortality: $P=0.428$ ).

\section{Discussion}

In this study, we found that in elderly patients with AMI, those who had higher inflammation-based scores at admission were associated with an increased incidence of in-hospital MACEs and mortality. Multiple logistic regression and ROC analysis indicated that the GPS had the best predictive value among the three score systems (GPS, PI and PNI) for elderly AMI patients.

Systemic inflammation is a well-known risk factor for atherosclerosis ${ }^{12}$ and plays an important role in atherosclerotic plaque instability. ${ }^{13}$ On the other hand,

Table 3 Multiple logistic regression analysis for in-hospital MACEs

\begin{tabular}{|c|c|c|c|c|c|c|}
\hline Variable & $\mathbf{R R}$ & $\begin{array}{l}\text { Univariate analysis } \\
95 \% \mathrm{Cl}\end{array}$ & $P$-value & $\mathbf{R R}$ & $\begin{array}{l}\text { Multivariate analysis } \\
95 \% \mathrm{Cl}\end{array}$ & $P$-value \\
\hline \multicolumn{7}{|l|}{ GPS } \\
\hline GPS (I vs 0 ) & 11.500 & $2.619-50.492$ & 0.001 & 6.711 & $1.409-31.968$ & 0.017 \\
\hline GPS (2 vs 0$)$ & 29.556 & $6.859-127.364$ & $<0.001$ & 14.063 & $3.018-65.535$ & 0.001 \\
\hline \multicolumn{7}{|l|}{$\mathrm{PI}$} \\
\hline $\mathrm{PI}$ (I vs 0) & 3.123 & $1.268-7.696$ & 0.013 & 2.594 & $0.758-8.885$ & 0.129 \\
\hline $\mathrm{PI}$ (2 vs 0 ) & 8.479 & $3.50 \mathrm{I}-20.537$ & $<0.001$ & 5.132 & $1.451-18.148$ & 0.011 \\
\hline LVEF & 0.928 & $0.905-0.952$ & $<0.001$ & 0.950 & $0.924-0.978$ & 0.001 \\
\hline Heart rate & 1.039 & $1.021-1.056$ & $<0.001$ & & & 0.432 \\
\hline Hemoglobin & 0.985 & $0.972-0.999$ & 0.030 & & & 0.634 \\
\hline Serum creatinine & 1.003 & $1.001-1.006$ & 0.015 & & & 0.452 \\
\hline Ig NT-proBNP & 7.229 & $3.674-14.224$ & $<0.001$ & 3.143 & I.443-6.848 & 0.004 \\
\hline Killip class & 5.066 & $2.749-9.338$ & $<0.001$ & & & 0.058 \\
\hline S2B within $12 \mathrm{~h}$ & 2.132 & $1.182-3.848$ & 0.012 & & & 0.770 \\
\hline
\end{tabular}

Note: a'Logistic regression analysis with forward stepwise regression model, adjusted for LVEF, heart rate, hemoglobin, serum creatinine, NT-proBNP, Killip class, symptomonset-to-balloon time.

Abbreviations: Cl, confidence interval; GPS, Glasgow Prognostic Score; LVEF, left ventricular ejection fraction; PI, Prognostic Index; S2B, symptom-onset-to-balloon time. 
Table 4 Multiple logistic regression analysis for in-hospital mortality

\begin{tabular}{|c|c|c|c|c|c|c|}
\hline Variable & HR & $\begin{array}{l}\text { Univariate analysis } \\
95 \% \mathrm{Cl}\end{array}$ & $P$-value & HR & $\begin{array}{l}\text { Multivariate analysis } \\
95 \% \mathrm{Cl}\end{array}$ & $P$-value \\
\hline \multicolumn{7}{|l|}{ GPS } \\
\hline GPS (I vs 0 ) & 17.111 & $2.230-131.291$ & 0.006 & 8.656 & $1.068-70.126$ & 0.043 \\
\hline GPS (2 vs 0$)$ & 22.892 & $3.009-174.17 \mid$ & 0.002 & 10.549 & $1.317-84.465$ & 0.026 \\
\hline \multicolumn{7}{|l|}{ PI } \\
\hline PI (I vs 0 ) & 1.696 & $0.608-4.728$ & 0.313 & & & 0.667 \\
\hline PI (2 vs 0$)$ & 4.823 & $1.850-12.575$ & 0.001 & & & 0.086 \\
\hline Age & 1.083 & $1.005-1.168$ & 0.036 & & & 0.123 \\
\hline LVEF & 0.941 & $0.915-0.969$ & $<0.001$ & 0.965 & $0.935-0.997$ & 0.031 \\
\hline Heart rate & 1.038 & $1.019-1.058$ & $<0.001$ & & & 0.247 \\
\hline SBP & 0.974 & $0.959-0.989$ & 0.001 & & & 0.061 \\
\hline Hemoglobin & 0.981 & $0.965-0.996$ & 0.015 & & & 0.290 \\
\hline Serum creatinine & 1.003 & $1.000-1.005$ & 0.044 & & & 0.137 \\
\hline Ig NT-proBNP & 4.346 & $2.079-9.086$ & $<0.001$ & & & 0.281 \\
\hline Killip class & 4.891 & $2.287-10.460$ & $<0.001$ & 3.072 & $|| 96-7.89 \mid$. & 0.020 \\
\hline S2B within $12 \mathrm{~h}$ & 2.335 & I.|I7-4.88| & 0.024 & & & 0.340 \\
\hline
\end{tabular}

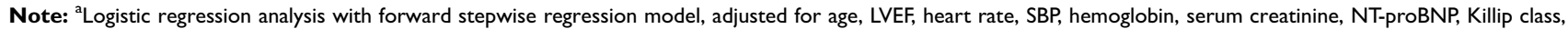
symptom-onset-to-balloon time.

Abbreviations: Cl, confidence interval; GPS, Glasgow Prognostic Score; LVEF, left ventricular ejection fraction; PI, prognostic index; SBP, systolic blood pressure; S2B, symptom-onset-to-balloon time.

inflammation is often a downstream pathological change of AMI, and excessive inflammation can lead to the aggravation of the original diseases, resulting in a vicious circle. In patients with AMI, several severe complications such as cardiac shock, ${ }^{14}$ heart failure ${ }^{15}$ and secondary infection

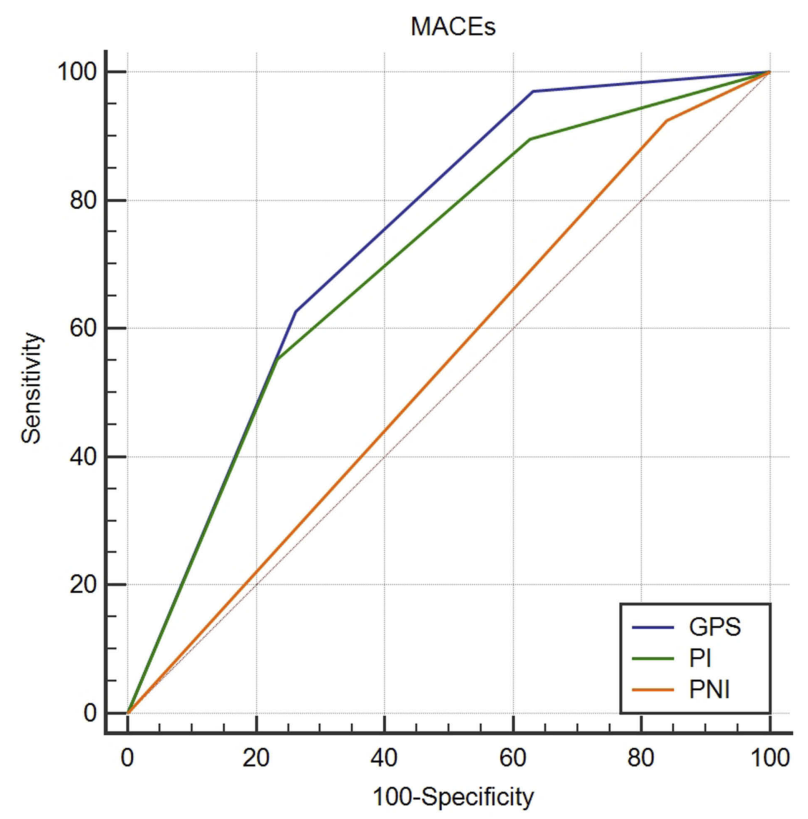

Figure 2 ROC curve of the inflammation-based prognostic scores for in-hospital MACEs.

Abbreviations: GPS, inflammation-based Glasgow Prognostic Score; PI, prognostic index; PNI, prognostic nutritional index; ROC, receiver-operating characteristic; MACE, major cardiovascular adverse event. can lead to increased inflammatory markers. Hence, higher inflammation-based scores at admission reflect the complex clinical baseline characteristics of patients. Meanwhile, severe inflammation can result in a larger infarction area ${ }^{16}$ and organ injury, ${ }^{17}$ which may lead to a worse outcome.

Our study was consistent with the work conducted by $\mathrm{Yu}$ Jia et $\mathrm{al}^{3}$. These authors also revealed a significant association between a higher GPS and mortality for STEMI patients, but the predictive value of the GPS in this study was lower than that in Yu's work (GPS-AUC: 0.677 vs 0.846). A possible explanation for the difference between these two studies may be that our study focused on elderly patients. As mentioned above, frailty and malnutrition are common in elderly people. Previous studies demonstrated that both frailty and malnutrition are associated with higher inflammatory parameters, such as CRP, interleukin-6 (IL-6) and lower serum albumin. ${ }^{7,18,19}$ There may be an overall increase in the GPS in elderly patients. Thus, the predictive value of a high GPS for poor prognosis may be decreased.

The power of the GPS for outcome prediction was stronger than that of the other two scoring systems in this study, which may be attributed to the combined effects of the components of the GPS. Serum albumin is an important parameter of the GPS score system. Recent studies have demonstrated that a decreased serum albumin concentration was associated with poor prognosis in acute 


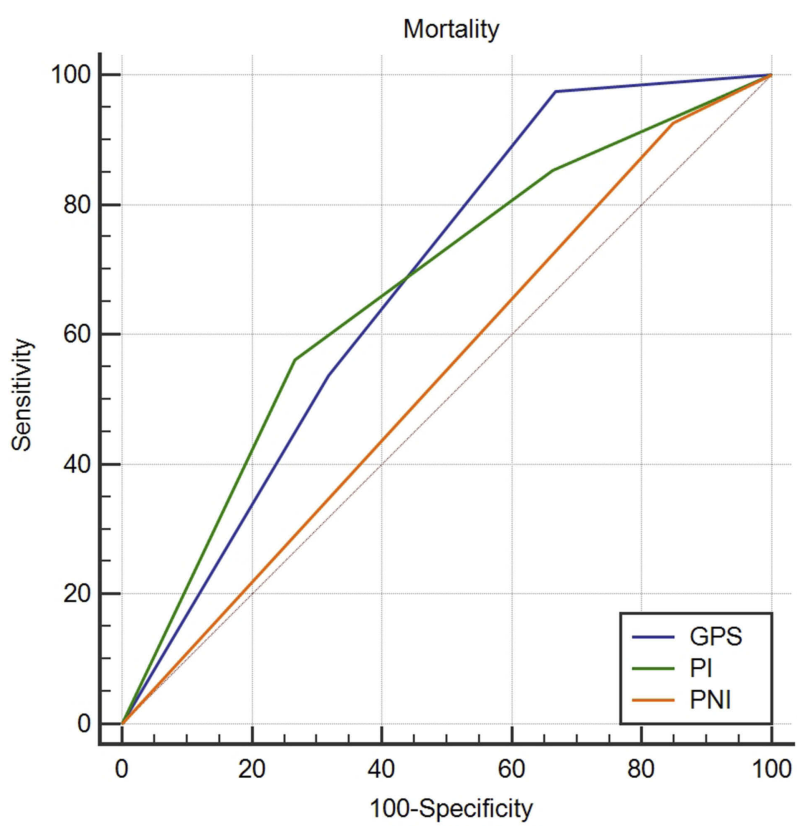

Figure 3 ROC curve of the inflammation-based prognostic scores for in-hospital mortality.

Abbreviations: GPS, inflammation-based glasgow prognostic score; PI, prognostic index; PNI, prognostic nutritional index; ROC, receiver-operating characteristic.

coronary syndromes. ${ }^{20,21}$ In the acute setting of AMI, inflammation can increase vascular permeability, which may increase the transfer of albumin out of the vascular compartment and result in a rapid decrease in the concentration of serum albumin. ${ }^{22}$ Meanwhile, hypoproteinaemia is also a manifestation of malnutrition. Decreased serum albumin is the result of the combined effects of inflammation and inadequate protein and caloric intake. ${ }^{22}$ Therefore, the GPS system reflects both systemic inflammatory reactions and nutritional status.

Traditional prognostic scores for acute coronary syndrome (ACS), such as the Global Registry of Acute Coronary Events

Table 5 Area under the ROC curve of prognostic scores for inhospital MACEs and mortality

\begin{tabular}{|l|l|l|l|l|}
\hline $\begin{array}{l}\text { Inflammation- } \\
\text { based scores }\end{array}$ & AUC & $\mathbf{9 5 \%} \mathbf{C l}$ & $\begin{array}{l}\text { P- } \\
\text { value }\end{array}$ & $\begin{array}{l}\text { P-value } \\
\text { (vs GPS) }\end{array}$ \\
\hline MACEs & & & & \\
GPS & 0.740 & $0.678-0.802$ & $<0.001$ & \\
PI & 0.703 & $0.634-0.773$ & $<0.001$ & $<0.001$ \\
PNI & 0.543 & $0.466-0.620$ & 0.293 & \\
\hline Mortality & & & & \\
GPS & 0.677 & $0.602-0.753$ & 0.000 & \\
PI & 0.667 & $0.577-0.757$ & 0.001 & 0.001 \\
PNI & 0.539 & $0.447-0.630$ & 0.428 & \\
\hline
\end{tabular}

Abbreviations: AUC, area under the curve; $\mathrm{Cl}$, confidence interval; GPS, Glasgow Prognostic Score; MACEs, major cardiovascular adverse events; PI, prognostic index; PNI, prognostic nutritional index; ROC, receiver-operating characteristic.
(GRACE) and Thrombolysis In Myocardial Infarction (TIMI) scores, do not consider inflammation-based components. Using traditional prognostic scores and inflammation-based prognostic scores in combination will be helpful for performing a comprehensive evaluation of patients with AMI.

There are some limitations in the present study. First, we only evaluated the in-hospital outcome of the patients. A long-term follow-up will provide a more thorough assessment of the predictive value of the inflammationbased prognostic scores. Second, this was a single centre study and may have selection bias. Finally, we did not record information on the medications that were used during hospitalization, which may have also influenced the outcome of the patients.

\section{Conclusion}

The severity of systemic inflammation is a strong predictor of poor prognosis in elderly patients with AMI. Among the three inflammation-based prognostic scores, the GPS has a better predictive value than the PI and PNI for in-hospital MACEs and mortality. Thus, the GPS score may represent a simple and useful tool for outcome prediction in elderly patients at the early stage of AMI.

\section{Acknowledgment}

The authors have no financial conflicts of interest to report.

\section{Disclosure}

The authors report no conflicts of interest in this work.

\section{References}

1. Ibanez B, James S, Agewall S, et al. 2017 ESC Guidelines for the management of acute myocardial infarction in patients presenting with ST-segment elevation. Rev Esp Cardiol (Engl Ed). 2017;70:1082.

2. Vanhaverbeke M, Veltman D, Pattyn N, et al. C-reactive protein during and after myocardial infarction in relation to cardiac injury and left ventricular function at follow-up. Clin Cardiol. 2018;41:1201-1206. doi: $10.1002 /$ clc. 23017

3. Jia Y, Li D, Cao Y, et al. Inflammation-based Glasgow Prognostic Score in patients with acute ST-segment elevation myocardial infarction: A prospective cohort study. Medicine (Baltimore). 2018;97: e13615. doi:10.1097/MD.0000000000013615

4. Zhang N, Zhu WL, Liu XH, et al. [A cross-sectional study on the association between frailty and muscular performances in hospitalized elder patients with coronary artery disease]. Zhonghua Nei Ke Za Zhi. 2019;58:265-269. doi:10.3760/cma.j.issn.0578-1426.2019.04.006

5. Valls T, Mach N. [Risk of malnutrition in people older than 75 years]. Med Clin (Barc). 2012;139:157-160. doi:10.1016/j.medcli.2012.01.029

6. Kim DY, Kim CO, Lim H. Quality of diet and level of physical performance related to inflammatory markers in community-dwelling frail, elderly people. Nutrition. 2017;38:48-53. doi:10.1016/j. nut.2016.12.023 
7. Hubbard RE, Woodhouse KW. Frailty, inflammation and the elderly. Biogerontology. 2010;11:635-641. doi:10.1007/s10522010-9292-5

8. Douglas E, McMillan DC. Towards a simple objective framework for the investigation and treatment of cancer cachexia: the Glasgow Prognostic Score. Cancer Treat Rev. 2014;40:685-691. doi:10.1016/ j.ctrv.2013.11.007

9. Kasymjanova G, MacDonald N, Agulnik JS, et al. The predictive value of pre-treatment inflammatory markers in advanced non-smallcell lung cancer. Curr Oncol. 2010;17:52-58. doi:10.3747/co. v17i4.567

10. Pinato DJ, North BV, Sharma R. A novel, externally validated inflammation-based prognostic algorithm in hepatocellular carcinoma: the prognostic nutritional index (PNI). $\mathrm{Br} J$ Cancer. 2012;106:1439-1445. doi:10.1038/bjc.2012.92

11. Thygesen K, Alpert JS, Jaffe AS, et al. Third universal definition of myocardial infarction. $\mathrm{J} \mathrm{Am}$ Coll Cardiol. 2012;60:1581-1598. doi:10.1016/j.jacc.2012.08.001

12. Hansson GK. Inflammation, atherosclerosis, and coronary artery disease. $N$ Engl $J$ Med. 2005;352:1685-1695. doi:10.1056/ NEJMra043430

13. Zairis MN, Lyras AG, Bibis GP, et al. Association of inflammatory biomarkers and cardiac troponin I with multifocal activation of coronary artery tree in the setting of non-ST-elevation acute myocardial infarction. Atherosclerosis. 2005;182:161-167. doi:10.1016/j. atherosclerosis.2005.01.039

14. Park M, Maciel AT, Noritomi DT, et al. Is persistent hypotension after transient cardiogenic shock associated with an inflammatory response? Braz J Med Biol Res. 2008;41:648-656. doi:10.1590/ s0100-879x2008000800002
15. Hartupee J, Mann DL. Positioning of inflammatory biomarkers in the heart failure landscape. J Cardiovasc Transl Res. 2013;6:485-492. doi:10.1007/s12265-013-9467-y

16. Yang M, Chen J, Zhao J, Meng M, Calvert J. Etanercept attenuates myocardial ischemia/reperfusion injury by decreasing inflammation and oxidative stress. PLoS One. 2014;9:e108024. doi:10.1371/journal.pone. 0108024

17. Liu H, Xiao X, Sun C, Sun D, Li Y, Yang M. Systemic inflammation and multiple organ injury in traumatic hemorrhagic shock. Front Biosci (Landmark Ed). 2015;20:927-933.

18. Carriere I, Dupuy AM, Lacroux A, Cristol J-P, Delcourt C. Biomarkers of inflammation and malnutrition associated with early death in healthy elderly people. J Am Geriatr Soc. 2008;56:840-846. doi:10.1111/j.1532-5415.2008.01677.x

19. Soysal P, Stubbs B, Lucato P, et al. Inflammation and frailty in the elderly: A systematic review and meta-analysis. Ageing Res Rev. 2016;31:1-8. doi:10.1016/j.arr.2016.08.006

20. Xia M, Zhang C, Gu J, et al. Impact of serum albumin levels on longterm all-cause, cardiovascular, and cardiac mortality in patients with first-onset acute myocardial infarction. Clin Chim Acta. 2018;477:89-93. doi:10.1016/j.cca.2017.12.014

21. Gonzalez-Pacheco H, Amezcua-Guerra LM, Sandoval J, et al. Prognostic implications of serum albumin levels in patients with acute coronary syndromes. Am J Cardiol. 2017;119:951-958. doi:10.1016/j.amjcard.2016.11.054

22. Don BR, Kaysen G. Serum albumin: relationship to inflammation and nutrition. Semin Dial. 2004;17:432-437. doi:10.1111/j.08940959.2004.17603.x
Clinical Interventions in Aging

\section{Publish your work in this journal}

Clinical Interventions in Aging is an international, peer-reviewed journal focusing on evidence-based reports on the value or lack thereof of treatments intended to prevent or delay the onset of maladaptive correlates of aging in human beings. This journal is indexed on PubMed Central, MedLine, CAS, Scopus and the Elsevier

\section{Dovepress}

Bibliographic databases. The manuscript management system is completely online and includes a very quick and fair peer-review system, which is all easy to use. Visit http://www.dovepress.com/ testimonials.php to read real quotes from published authors. 